\title{
EOLA1 inhibits lipopolysaccharide-induced vascular cell adhesion molecule- 1 expression by association with MT2A in endothelial cells
}

\author{
Min Luo ${ }^{1}$, Weiling Len ${ }^{1}$, Xiaotian Lei ${ }^{1}$, Hao Meng ${ }^{1}$, Xinshou Ouyang ${ }^{2}$ and Ziwen Liang*1 \\ ${ }^{1}$ Department of Endocrinology, Southwest Hospital of Third Military Medical University, Chongqing, China \\ ${ }^{2}$ Section of Digestive Diseases, Department of Internal Medicine, Yale University School of Medicine, New Haven, CT, USA
}

\begin{abstract}
Endothelial-overexpressed lipopolysaccharide-associated factor 1 (EOLA1), an unspecified human gene (GenBank No. AY074889), has been identified pioneerly by our research group as the one of lipopolysaccharide (LPS) responsive genes in human umbilical vein endothelial derived cell. Our previous studies have indicated metallothionein 2A (MT2A) as the associated protein, and an importance of EOLA1 but less understood roles during LPS-induced inflammatory response. In this report, we determined the subcellular localization of EOLA1, and the regulatory capacity of EOLA1 on vascular cell adhesion molecule-1 (VCAM-1) in ECV304 cells stimulated by LPS. Our results showed that EOLA1 is broadly diffuse in the cells. LPS significantly induced EOLA1 and MT2A expression, and surprisingly knockdown of EOLA1 in ECV304 cells significantly enhanced LPS-induced VCAM-1 production. In consistent with this, overexpression of EOLA1 reduced the LPS-induced VCAM-1 production. Furthermore, knockdown of MT2A reduced LPS-induced VCAM-1 production. Collectively, our results demonstrate a negative regulatory role of EOLA1 on LPS-induced inflammation response under the possible association with MT2A in endothelial cells.
\end{abstract}

\section{Introduction}

Endothelial-over-expressed lipopolysaccharide-associated factor 1 (EOLA1, GenBank No.074889, also named as CXorf40A) has been identified by our research group from genes with differential expression after stimulation of an umbilical vein endothelial cell line ECV304 with lipopolysaccharide (LPS) in 2004 [1,2]. However, its biological function is less understood. EOLA1 is located at human chromosome Xq28, and contains 6,248 bases in genomics and 5 exons separated by 4 introns. The EOLA1 gene is conserved in chimpanzee, rhesus monkey, dog, mouse, rat, chicken, zebrafish, and frog. EOLA1 protein comprises 158 amino acids and has several glycosylation and phosphorylation sites and the helix-turn-helix motif without signal peptide and membrane spanning domain. These structural features indicate that EOLA1 is an intracellular protein and may interact with relevant proteins in the cytoplasm and nucleus to regulate cell function. The bioinformatics analysis reviewed that EOLA1 gene belongs to activating signal cointegrator 1 (ASC-1) homology (ASCH) superfamily. ASCH, a small beta-barrel domain, is frequently observed in many organisms, although its function has not yet been clearly defined [3]. ASCH has been proposed to be functional as an RNA-binding domain during coactivation, RNA-processing and the regulation of prokaryotic translation [4].

EOLA1 is slightly expressed under the steady condition, and its expression is significantly increased in response to LPS in ECV304 cells. The protein association between EOLA1 and metallothionein 2A (MT2A) has been confirmed by yeast double-hybridization and co-immunoprecipitation assay [2,5]. MT2A is known to have antiinflammatory, anti-endotoxin, and tumor-inhibiting effects in cell cultures [6,7]. In addition, MT2A inhibits cell growth through the induction of apoptosis and G2/M arrest, which negatively regulates NF- $\kappa B$ pathway by up-regulation of I $\kappa \mathrm{B}-\alpha$ and down-regulation of $\mathrm{p}-\mathrm{I} \kappa \mathrm{B}-\alpha$ and cyclin D1 [8]. Based on a rat liver transplantation model, we have found significant changes of EOLA1 expression in transplantation rejection [9]. These results suggest a possible role of EOLA1 in transmitting inflammation-related signals through association with MT2A and thus participate the regulation of inflammatory response in endothelial cells.

LPS are the major outer surface membrane components present in almost all Gram-negative bacteria and act as ligand in triggering innate immune response via specific receptors in diverse eukaryotic species ranging from insects to humans $[10,11]$. The induction of vascular cell adhesion molecule-1 (VCAM-1) is the hallmark of activated vascular endothelial cell [12]. VCAM-1 that belongs to the immunoglobulin superfamily, regulates leukocyte recruitment and immune response to sites of inflammation, and accelerates the development of atherosclerosis $[13,14]$. While it is known that LPS induces VCAM-1 expression in vascular endothelial cells.

To understand the biological function of EOLA1 in regulating inflammation in a broad spectrum, we examined the subcellular distribution, and the efficacy of EOLA1 in the regulation of LPS induced inflammatory gene response including VCAM-1 in ECV304 cells.

Correspondence to: Ziwen Liang, Department of Endocrinology, Southwest Hospital of Third Military Medical University, Chongqing, China; E-mail: ziwenliang99@163.com

Received: November 01, 2015; Accepted: November 18, 2015; Published: November 21, 2015 


\section{Materials and methods}

\section{LPS preparation and cell culture}

Purified native LPS phenol (Sigma) was dissolved in phosphatebuffered saline (PBS) to the dilution of $1 \mathrm{mg} / \mathrm{ml}$ and stored at $-20^{\circ} \mathrm{C}$. For experiments, the LPS stock solution was further diluted into supplemented tissue culture media to the working concentration of 100 $\mathrm{ng} / \mathrm{ml}$. ECV304 (ATCC) were cultured in Medium 199 supplemented with $10 \%$ fetal bovine serum, $3 \mathrm{mg} / \mathrm{ml}$ Hepes, $100 \mathrm{U} / \mathrm{ml}$ penicillin G, $100 \mathrm{mg} / \mathrm{ml}$ streptomycin and $6 \mathrm{U} / \mathrm{L}$ insulin on gelatin coated dishes in a humidified atmosphere of $5 \% \mathrm{CO}_{2}$. Confluent cultures were detached with EDTA-Trypsin solution and split into 1:3 for further use.

\section{Construction of EGFP-EOLA1 fusion protein expression plasmid and cell transfection}

A pair of primers was synthesized according to the open reading frame (ORF) sequence of EOLA1 (forward primer: 5'-ACCAGGATCCTCTCTTCATGCCCAAAG-3', reverse primer: 5'AGCAGGATCCTCTCTTCATGCCCCAAAG-3'); $E c o R \quad I$ and $\mathrm{BamH}$ I endonuclease sites were introduced into the terminals of forward and reverse primers, respectively. ECV304 cells were stimulated with LPS $(100 \mathrm{ng} / \mathrm{ml})$ for 6 hours, and then the total RNA was extracted for RT-PCR (TaKaRa), and finally the ORF sequence of EOLA1 was amplified. The PCR product and pEGFP-N2 plasmid (Clontech) were cut with endonucleases EcoR I and BamH I, then the enzyme cutting products were recovered by electrophoresis and linked with DNA ligase (TaKaRa), and finally the target ORF sequence was inserted into pEGFP-N2 plasmid and single colonies were selected for sequencing (TaKaRa) after transformation of competent Escherichia coli DH5a.

For cell transfection, ECV304 cells were split onto 6-well culture plate (pre-covered with a sterile coverslip) by $1.5 \times 10^{5} /$ well. When $80 \%$ fusion was achieved in the cell culture, the fusion protein expression plasmid pEGFP-EOLA1 and the control plasmid pEGFP-N2 were transfected into cells using the liposome Lipofectamine ${ }^{\mathrm{TM}} 2000$ (Invitrogen) for 48 hours. The transfection efficiency was determined by GFP positive versus negative cells.

\section{Subcellular distribution of EOLA1}

After cell transfection for 48 hours, the culture medium was removed, and then the cells were rinsed twice with PBS and fixed with $4 \%$ PFA. A part of cells was used to examine the subcellular location; the cells were incubated with rat anti-GFP antibody (primary antibody) and goat anti-rat antibody (secondary antibody), stained with DAPI, and photographed under LSM510 META laser confocal microscope (Zeiss). For observation under immunoelectron microscope, the cells were fixed with $4 \%$ PFA, and then oxidized with 3\% hydrogen dioxide and incubated with serum. The incubation was performed with the previously prepared rabbit anti-EOLAl polyclonal antibody as primary antibody and HRP-labeled goat anti-rabbit IgG antibody as secondary antibody. Then the cells were stained with HRP-labeled streptoantibiotin and diaminobenzidine (DAB), re-fixed with $2.5 \%$ glutaral and fixed with $0.1 \%$ osmic acid, dehydrated with acetone at a gradient, and then preserved overnight in the saturated uranyl acetate prepared with $70 \%$ acetone. The cells were further rinsed with pure acetone, soaked with the mixture of acetone and epoxy resin (v:v=1:1), treated with pure embedding medium (without epoxy resin curing promoter DMP-30) and embedding medium (with DMP-30) at $40^{\circ} \mathrm{C}$, and embedded with epoxy resin 618 . Finally the cells were made into ultrathin sections, stained with lead, observed and photographed under TECNA110transmission electron microscope (Philip).

\section{Knock-down experiments}

Knock-down of EOLA1 was accomplished using RNA interfere (RNAi). The oligonucleotide sequences of EOLA1-shRNA (small hairpin RNA) were sense: 5'-AGGAGGCAAGGATGTATT CTTCAAGAGAGAATACATCCTTGTTTTTTT-3', and anti-sense: 5' - AATTAAAAAAAGGAGGCAAGGATGTATTCTCTCTT GAAGAATACATCCTTGCCTCCTGGCC-3'. For knock-down of MT2A, the oligonucleotide sequences of MT2A-shRNA were sense: 5'-GATCCGCCGTG ACCGTTTGCTATATTTCAAGAGAATATAGCAAACGGTCACGGTTTTTTGGAAA-3', and anti-sense: 5'-AGCTTTCCAAAAAACCGTGACCGTTTGCTATATTCTCTTGAAATATAGCAAACGGTCACGGCG-3'. The shRNA expression cassette driven by $\mathrm{H} 1$ promoter was engineered by inserting those oligonucleotides into the plasmid pSilencer 3.1/H1 hygro (Ambion). An unengineered plasmid, $\mathrm{pH} 1$ was used as a blank control. The cells were transfected with $\mathrm{pH} 1, \mathrm{pH} 1-\mathrm{EOLA} 1$ shRNA, or pH1-MT2A shRNA with lipofectmine 2000 in serum-free OPTI-MEMI (Invitrogen) according to the instruction manual. The concentration of DNA vectors for transfection was $8 \mu \mathrm{g} / \mathrm{ml}$ in a final volume of $500 \mu \mathrm{l}$. The cells were washed after $4 \mathrm{~h}$ incubation and resuspended in complete growth medium with serum for further experiments.

\section{RT-PCR and VACM-1 assay}

The Total RNA was isolated from the cells using Trizol reagent following the manufacturer's instructions (Invitrogen). To measure the gene mRNA expression level, RT-PCR was performed using one step reagent kit (TaKaRa). The primers are synthesized from commensal (EOLA1 upstream: 5'-GCTCGAATTCATGAAGTTTGGCTGCCTCTC-3', downstream: 5'-AGCAGGATCCTCTCTTCATGCCCCAAAG-3'. $\beta$-actin: upstream: 5'-CGGGAAATCGTGCGTGACATT-3', downstream: 5'-CTAGAAGCATTTGCGGTGGAC-3'). For VACM-1 assay, the cells were homogenized in PBS (1:2, w/v) containing $1 \%$ protease inhibitors and then centrifuged at $12,000 \times \mathrm{g}$ for 20 $\min$ at $4^{\circ} \mathrm{C}$. The supernatants were analyzed using ELISA kit (Sigma) according to the manufacturer's instructions.

\section{Western blot analysis}

Transfected cells were lysed with RIPA buffer supplemented with Complete Protease Inhibitor Tablets (Roche). Lysate protein concentration was measured by Lowry assay. The protein samples (30 $\mu \mathrm{g})$ were loaded on SDS-9\% polyacrylamide gels and then transferred to Protein nitrocellulose membranes in an electroblotting apparatus, using standard procedures. Immunodetection was performed as described previously [2] by using anti-EOLA1, anti-MT2A or anti-GFP as first antibody, and an second antibody Ig-horseradish peroxidase conjugate (Invitrogen), anti- $\beta$-actin as a loading control. The antiEOLA1 polyclonal antibody was produced from New Zealand white rabbits immunization and identified by Western blot. IgGs against EOLA1 were purified on a protein A Sepharose column. The efficiency of polyclonal antibody was measured by ELISA analysis.

\section{Statistical analysis}

Data were expressed as means \pm standard error. Stuent's $t$ tests were performed to determine the statistical significance for the assays of ELISA; $p<0.05$ was considered statistically significant. 


\section{Results}

\section{Subcellular distribution of EOLA1in ECV304 cells}

ECV304 cells were transfected with pEGFP-EOLA1 fusion protein expression plasmid and the control of pEGFP-N2 plasmid for $48 \mathrm{~h}$. The images were observed under laser confocal microscope. ECV304 cells transfected with either control plasmid or fusion protein expression plasmid demonstrated uniform whole-cell distribution by green fluorescence, while the cells transfected with fusion protein expression plasmid also had nuclear aggregation (Figure 1A). With the prepared rabbit anti-EOLA1 polyclonal antibody as primary antibody, the transfected cells were labeled immunologically and then observed under electron microscope. There was no immunological precipitate in the cells transfected with control plasmid but significantly immunological precipitates deposited in the cytoplasmic ground substance of cells transfected with fusion protein expression plasmid (Figure 1B).

\section{LPS induction of EOLA1 expression and VCAM-1 production in ECV304 cells}

ECV304 cells were grown to $80 \%$ confluence and stimulated with LPS $(100 \mathrm{ng} / \mathrm{ml})$ for a time-course as indicated. The cells showed that EOLA1 transcription was increased in a time-dependent manner (Figure 2A). VCAM-1 concentrations in the clarified culture supernatants were then measured by ELISA. Exposure of the cells to LPS $(100 \mathrm{ng} / \mathrm{ml})$ induced significantly increase of VCAM-1 production (Figure 2B).

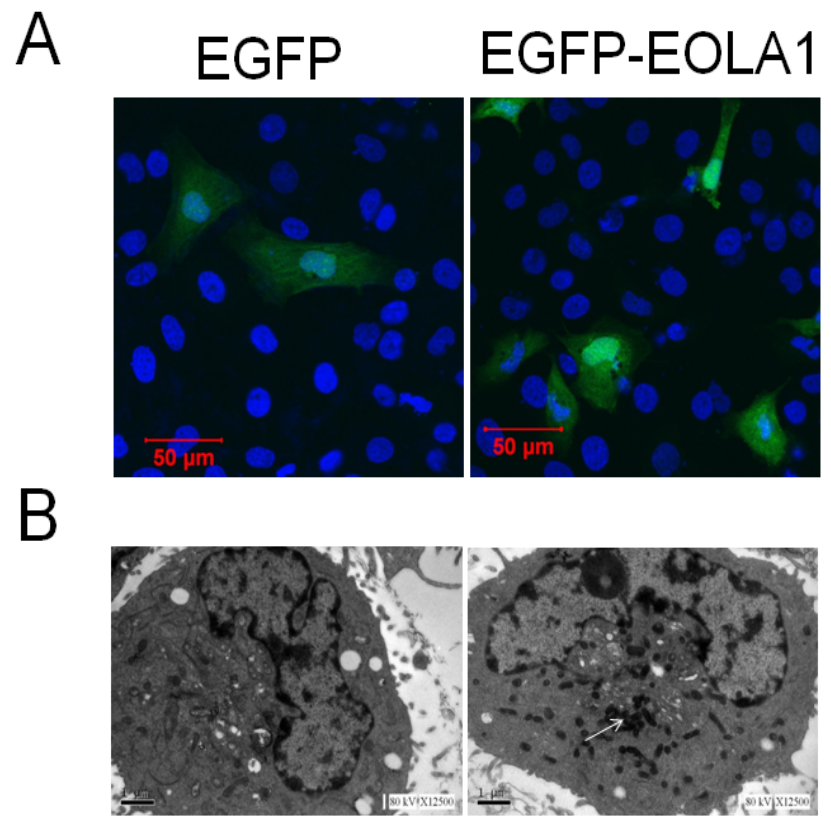

Figure 1. Subcellular location of EOLA1 is indicated in ECV304 cells. ECV304 cells were transfected with the blank plasmid pEGFP-N2 and the fusion protein expression plasmid pEGFP-EOLA1. After 48 hours culture, the transfected cells were fixed with $4 \%$ PFA. A, The cells were incubated with rat anti-GFP antibody (primary antibody) and goat antirat antibody (secondary antibody), stained with DAPI, and photographed under LSM510 META laser confocal microscope. B, The cells were incubated with rabbit anti-EOLAl polyclonal antibody (primary antibody) and HRP-labeled goat anti-rabbit IgG antibody (secondary antibody), stained with HRP-labeled strepto-antibiotin and diaminobenzidine, and embedded with epoxy resin 618. Finally the cells were made into ultrathin sections, stained with lead, observed and photographed under TECNA110transmission electron microscope. The white arrow identifies immune sediment deposition in ECV304 cells transfected with pEGFP-EOLA1 by anti-EOLA1 antibody.
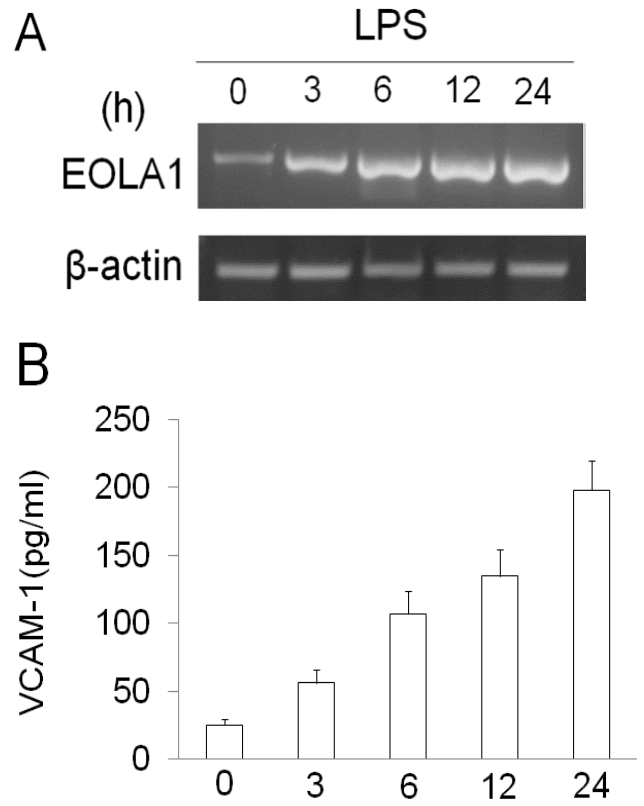

(h)

Figure 2. LPS induces the production of EOLA1 and VCAM-1 in ECV304 cells. A, ECV304 cells were treated with LPS $(100 \mathrm{ng} / \mathrm{ml})$ for various periods of time $0,3,6,12$, and 24 hours and the total RNAs were analyzed by RT-PCR to EOLA1. B, ECV304 cells were stimulated with LPS $(100 \mathrm{ng} / \mathrm{ml})$ for the indicated periods of time and the supernatants were collected and assayed for VCAM-1production by ELISA. Data show one representative experiment out of three independent experiments.

\section{Overexpression of EOLA1 inhibited the expression of VCAM- 1 induced by LPS in ECV304 cells}

To further characterize the functional effects of EOLA1 on VCAM-1 induction in ECV304 cells, the cells were co-transfected with pOPRSV I -EOLA1-EGFP and pCMV-Lac I plasmids to stably overexpress EOLA1 after induced by IPTG (Figure 3A). The cells with overexpression of EOLAl were treated with LPS $(100 \mathrm{ng} / \mathrm{ml})$ after adding IPTG for $48 \mathrm{~h}$. The VCAM-1 protein production is cell supernatant was detected by ELISA assay in $6 \mathrm{~h}$ after LPS treatment. The results showed that LPS significantly induces VCAM- 1 protein secretion, and overexpression of EOLA1 decreased the VCAM-1 protein level induced by LPS in ECV304 cells (Figure 3B).

Knock-down EOLA1 enhances the expression of LPS induced VCAM-1 production in ECV304 cells

To further investigate the efficacy of EOLA1 on LPS induced VCAM-1 production in ECV304 cells, the cells were transfected with EOLA1 shRNA to silence EOLA1 gene. EOLA1 expression was confirmed significantly down-regulated in EOLA1-shRNA transfected cells by western blot. Knock-down EOLA1 also shows inhibition on the expression of MT2A (Figure 4A). Knock down EOLA1 enhances LPS induced VCAM-1 protein secretion (Figure 4B).

\section{EOLA1 regulates VCAM-1 production through MT2A}

EOLA1 has an association with MT2A in ECV304 cells, and EOLA1 regulates the expression of MT2A, suggesting EOLA1 mediates VCAM-1 production through association with MT2A. We further investigated the roel of MT2A in LPS induced immune response. MTA2 knockdown cells were treated with LPS, and the cell supernatant 

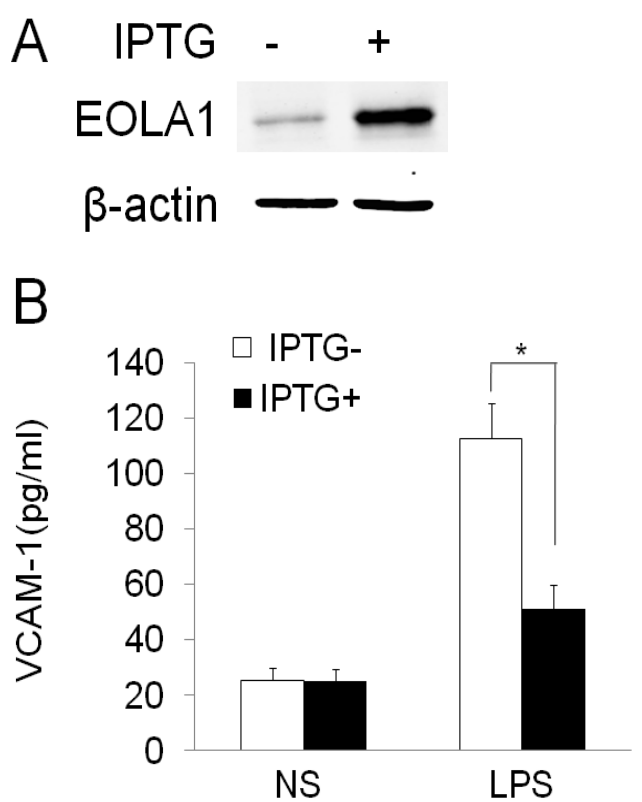

Figure 3. Overexpression of EOLA1 prevents LPS induced VCAM-1 production in ECV304 cells. ECV304 cells were transcript with pOPRSV I -EOLA1-EGFP and pCMVLac I plasmids, and induced by IPTG. A, Overexprssion of EOLA1 was demonstrated by Western blotting analysis with anti-EOLA1 antibody 48 after IPTG induced. B, The induced cells with IPTG were tested for their ability to produce VCAM-1 upon LPS stimulation $(100 \mathrm{ng} / \mathrm{ml})$ for $6 \mathrm{~h}$ using a commercial ELISA kit. Data represent the mean \pm S.D of three independent experiments. ${ }^{*} \mathrm{p}<0.05$.
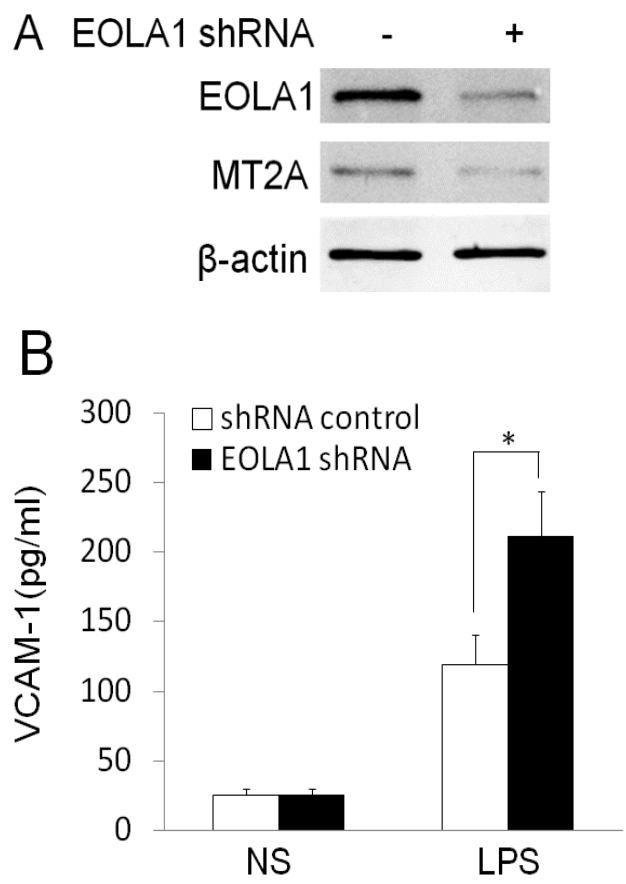

Figure 4. Knock-down of EOLA1 with shRNA inhibits the expression of MT2A and increases the LPS-induced VCAM-1 production in ECV304 cells. A, ECV304 cells were transfected with control or EOLA1 shRNA for 48 hours. The cells were lysed and analyzed by Western blotting with anti-EOLA1 or anti-MT2A antibodies, $\beta$-actin as a loading control. B, ECV304 cells were transfected with control or EOLA1 shRNA for 24 hours and then cultured with or without LPS $(100 \mathrm{ng} / \mathrm{ml})$ and the concentration of VCAM-1 in the culture supernatants was determined 6 after stimulation. Data represent the mean \pm S.D of three independent experiments. ${ }^{*} \mathrm{p}<0.05$. of VCAM-1 was measured by ELISA assay in 6h after LPS treatment. The results showed that knock-down MT2A down regulates LPS induced VCAM-1 protein secretion in ECV304 cells (Figure 5).

\section{Discussion}

EOLA1 is a novel gene pioneerly identified by our research group from the screening of LPS induced gene expression in ECV304 cells. However, little is known about its biological function in inflammation. To understand the subcellular distribution of EOLA1, we directionally cloned the ORF sequence of EOLA1 into pEGFP-N2 carrier and thus successfully constructed the eukaryotic expression plasmid of EGFP-EOLA1 fusion protein to transfect ECV304 cells. Furthermore, we observed the transfected cells under laser confocal microscope after transient expression. The observations showed strong green fluorescence signals in both the cytoplasm and nucleus. The DAPI staining confirmed that the fusion protein was expressed in the cytoplasm and aggregated in the nucleus. Besides, we further observed the subcellular localization of EOLA1 at a level of cell ultrastructure by the use of immunoelectron microscopic enzyme labeling technique, and the results confirmed the expression of EOLA1 in the cytoplasm. The above observations indicate that EOLA1 is an intracellular protein and has a capacity to translocate into the nucleus from the cytoplasm, although such phenomenon still needs to be further confirmed in the future.

ECV304 cells belong to human umbilical vein endothelial cells, which can be activated by LPS stimulation. After LPS stimulated cells, signal transduction through membrane receptor Toll-like receptor-4, has been relieved I- $\kappa B$ inhibition of NF- $\kappa B$, which turns into the nucleus, promoted the expression of VCAM-1 and release, activate
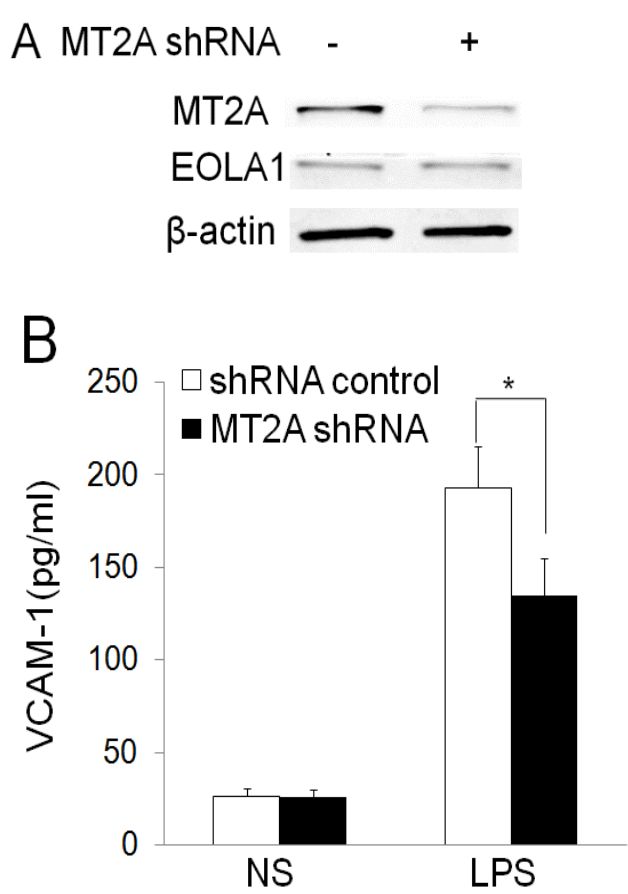

Figure 5. EOLA1 mediates LPS induced VCAM-1 production by MT2A. A, ECV304 cells were transfected with MT2A shRNA or control shRNA, and the cells were lysed and analyzed by Western blotting with anti-MT2A or ant-EOLA1 antibody, $\beta$-actin as a loading control. B, ECV304 was transfected with MT2A shRNA or control shRNA and then stimulated with LPS $(100 \mathrm{ng} / \mathrm{ml})$ for $6 \mathrm{~h}$. VCAM-1 production was assayed by ELISA. Data represent the mean+S.D of three independent experiments. ${ }^{*} \mathrm{p}<0.05$. 
endothelial cells and attract mononuclear macrophage accumulation, induce inflammatory response $[15,16]$. Recent study also showed that LPS-induced VCAM-1 expression contributes to the initiation of the atherosclerotic process $[17,18]$. Our experiments showed that LPS induces EOLA1 expression along with VCAM-1 induction, overexpression of EOLA1 reduces LPS induced the production of VCAM-1 in ECV304 cells, and depletion of EOLA1 significantly enhances LPS induced the production of VCAM-1. Our results suggest that EOLA1 controls the degree of endothelial cell activation by LPS, and possibly negative regulates the inflammatory immune response.

MT2A is a low-molecular-weight protein in the cytoplasm, plays multiple roles in cell metabolism, and has the effects on antiinflammation, anti-endotoxin and inhibiting tumor growth. Recently it has been found that MT2A reduces cell inflammatory response by inhibiting the activation of NF- $\kappa \mathrm{B}$, through up-regulating I $\mathrm{KB}-\alpha$. Our results show that EOLA1 regulates the expression of MT2A and affects the production of VCAM-1 by association with MT2A, therefore the excessive activation of ECV304 cells could be avoided, and the fine regulation and timely control of inflammatory reaction could be thereby achieved. This will avoid the damage of excessive inflammatory reaction to tissues and cells. The detail mechanism of EOLA1 association with MT2A and their functional effects on the NF$\kappa \mathrm{B}$ signaling pathway needs to be further characterized.

In conclusion, EOLA1 plays a negative regulatory role on LPSinduced inflammation response under the association with MT2A in ECV304 cells.

\section{Conflict of Interests}

The authors declare that they have no competing interests.

\section{Acknowledgments}

This work was supported by research grants from the National Natural Science Foundation of China (Grants No. 30670838).

\section{References}

1. Ziwen L, Xiangdong L, Zongcheng Y (2003) Cloning of differentially expressed genes following lipopolysaccharides stimulation in human umbilical vein endothelial cells. Chinese J Traumatol 6: 107-113.

2. Liang Z, Yang Z (2004) Identification and characterization of a novel gene EOLA1 stimulating ECV304 cell proliferation. Biochem Biophys Res Commun 325: 798-802. [Crossref]

3. Park SY, Park JH, Kim JS (2011) Cloning, expression, purification, crystallization and preliminary X-ray diffraction analysis of an ASCH domain-containing protein from Zymomonas mobilis ZM4. Acta Crystallographica Section F-structural Biology
Communications 67: 310-312. [Crossref]

4. Iyer LM, Burroughs AM, Aravind L (2006) The ASCH superfamily: novel domains with a fold related to the PUA domain and a potential role in RNA metabolism. Bioinformatics 22: 257-263. [Crossref]

5. Liu Y, Liu H, Chen W, Yang T, Zhang W (2014) EOLA1 protects lipopolysaccharide induced IL-6 production and apopotosis by regulation of MT2A in human umbilical vein endothelial cells. Mol Cell Biochem 395: 45-51. [Crossref]

6. Albrecht EA1, Dhanasekaran SM, Tomlins S (2011) Immediate early inflammatory gene responses of human umbilical vein endothelial cells to hemorrhagic venom. Inflamm Res 60: 213-217. [Crossref]

7. Pérez MJ, Cederbaum AI (2003) Metallothionein $2 \mathrm{~A}$ induction by zinc protects HEPG2 cells against CYP2E1-dependent toxicity. Free Radic Biol Med 34: 443-455. [Crossref]

8. Pan Y, Huang J, Xing R, Yin X, Cui J, et al. (2013) Metallothionein 2A inhibits NF$B$ pathway activation and predicts clinical outcome segregated with TNM stage ingastricancer patients following radical resection. J Transl Med 11: 173-187. [Crossref]

9. Ran CF, Dou K, Liang Z, Liu Y, Li K (2008) Changes in the expression of endothelialoverexpressed lipopolysaccharide-associated factor 1 in grafts during acute rejection following liver transplantation in rats. $J$ Int Med Res 36: 460-466. [Crossref]

10. Rice JB, Stoll LL, Li WG (2013) Low-level endotoxin induces potent inflammatory activation of human blood vessels: inhibition by statins. Arterioscler Thromb Vasc Biol 23:1576-1582.

11. Stoll LL, Denning GM, Weintraub NL (2004) Potential role of endotoxin as a proinflammatory mediator of atherosclerosis. Arterioscler Thromb Vasc Biol 24: 2227 2236. [Crossref]

12. Liu B, Cheng L, Liu D, Wang J, Zhang X, et al. (2012) Role of p38 mitogen-activated protein kinase pathway in Porphyromonas gingivalis lipopolysaccharide-induced VCAM-1expression in human aortic endothelial cells. J Periodontol 83: 955-962. [Crossref]

13. Cybulsky MI, Ilyama K, Li H, (2001) "A major role for VCAM-1, but not ICAM-1, in early atherosclerosis," Journal of Clinical Investigation 107: 1255-1262. [Crossref]

14. Ley K, Huo Y (2001) "VCAM-1 is critical in atherosclerosis". J Clin Invest 107: 12091210. [Crossref]

15. Guijarro-Muñoz I, Compte M, Álvarez-Cienfuegos A, Álvarez-Vallina L, Sanz L (2014) Lipopolysaccharide activates Toll-like receptor 4 (TLR4)-mediated NF-kB signaling pathway and proinflammatory response in human pericytes. J Biol Chem 289: 2457-2468. [Crossref]

16. Xu Y, Xu X, Jin H, Yang X, Gu Q, et al. (2013) Effects of a thrombomodulin-derived peptide on monocyte adhesion and intercellular adhesion molecule-1 expression in lipopolysaccharide-induced endothelial cells. Mol Vis 19: 203-212. [Crossref]

17. Shan Y, Lin N, Yang X, Tan J, Zhao R, et al. (2012) Sulphoraphane inhibited the expressions of intercellular adhesion molecule-1 and vascular cell adhesion moleculethrough MyD88-dependent toll-like receptor-4 pathway in cultured endothelial cells. Nutr Metabol Cardiovas Dise 22: 215-222. [Crossref]

18. Liu B, Wang J, Cheng L, Liang J (2013) Role of JNK and NF-kB pathways in Porphyromonas gingivalis LPS-induced vascular cell adhesion molecule-1 expression in human aortic endothelial cells. Mol Med Rep 8:1594-1600. [Crossref]

Copyright: (C2015 Luo M. This is an open-access article distributed under the terms of the Creative Commons Attribution License, which permits unrestricted use, distribution, and reproduction in any medium, provided the original author and source are credited. 